\title{
Performance Assessment of an Artificial Immune System Multiobjective Optimizer by Two Improved Metrics
}

\author{
Maoguo Gong \\ Xidian University \\ Institute of Intelligent Information \\ Processing, PO Box 224, Xidian \\ University, Xi'an, 710071, China \\ +86-02988209786 \\ Maoguo_Gong@hotmail.com \\ Ronghua Shang \\ Xidian University \\ Institute of Intelligent Information \\ Processing, PO Box 224, Xidian \\ University, Xi'an, 710071, China
}

\author{
Licheng Jiao \\ Xidian University \\ Institute of Intelligent Information \\ Processing, PO Box 224, Xidian \\ University, Xi'an, 710071, China \\ +86-029 88201023 \\ LCJiao@mail.xidian.edu.cn
}

\author{
Haifeng Du \\ Xi'an Jiaotong University \\ School of Mechanical Engineering, \\ Xi'an Jiaotong University, Xi'an, \\ 710049, China \\ Haifengdu72@163.com
}

\begin{abstract}
In this study, we introduce two improved assessment metrics of multiobjective optimizers, Nondominated Ratio and Spacing Distribution, and analyze their rationality and validity. Based on the concept of Immunodominance and Antibody Clonal Selection Theory, a novel multiobjective optimization algorithm, Immune Dominance Clonal Multiobjective Algorithm (IDCMA), is put forward. The simulation comparisons between IDCMA and the Strength Pareto Evolutionary Algorithm show that IDCMA has the best performance in popular metrics such as Spacing, Coverage of Two Sets and the two new metrics presented in this paper when lowdimensional multiobjective problems are concerned. The statistical results of the four metrics also show that Spacing Distribution conquers some limitations of Spacing triumphantly, and Nondominated Ratio conquers the limitation of Coverage of Two Sets that only compared between two sets.
\end{abstract}

\section{Categories and Subject Descriptors}

\section{F.2.1 [Analysis of Algorithms and Problem Complexity]:}

Numerical Algorithms and Problems - Number-theoretic computation

General Terms: Algorithms, Measurement, Performance, Design.

Keywords: multiobjective optimization, performance assessment, immune dominance, clonal selection, artificial immune system.

\section{INTRODUCTION}

A difficulty in multiobjective optimization is the quantitative comparison of the performance of different algorithms. To assess the output sets quantificationally is also a research focus going with the

Copyright is held by the author/owner(s).

GECCO'05, June 25-29, 2005, Washington, DC, USA.

ACM 1-59593-010-8/05/0006. research of new algorithms. Zitzler and Van Veldhuizen et al have proposed a lot of metrics of performance assessment and provided some performance comparisons of representative multiojective evolutionary algorithms respectively [4][5].

Artificial immune system (AIS) makes use of the mechanism of vertebrate immune system, and constructs new intelligent algorithms with immunology terms and fundamental, for providing some novel methods to solve problems [1]. Its research production refers to many fields like control, data processing, optimization learning and trouble diagnosing, and it has been a research hot spot after the neural network, fuzzy logic and evolutionary computation [1].

\section{TWO IMPROVED ASSESSMENT METRICS OF MOEAS}

Generally, a multiobjective optimization problem has a set of Pareto-optimal solutions, instead of one single optimal solution, so the goal in a multiobjective optimization is different from that in a single-objective optimization. In multiobjective optimization, we desire to find as many different Pareto-optimal or near Paretooptimal solutions as possible. Generally speaking, multiobjective optimization includes three objectives, minimal distance to the Pareto-optimal front, and good distribution, and maximum spread. Quantitative performance assessments of MOEAs should take all of the three objectives into account. In the references [3] [4] and [5], some quantitative metrics have been presented for the performance assessment of multiobjective optimization, such as Dominated Space, Coverage of Two Sets, Hyperarea Ratio, Spacing, and so on.

In this paper, a new metric $\varphi$ called Spacing Distribution is proposed, and it can be defined as follows by modifying the metric Spacing.

Definition 1 (Spacing Distribution) Let $\boldsymbol{X}^{\prime} \subseteq \boldsymbol{X}$ be a set of decision vectors. The function $\varphi$

$$
\varphi \square \sqrt{\frac{1}{\left|X^{\prime}\right|-1} \sum_{i=1}^{\left|X^{\prime}\right|}\left(\bar{d}-d_{i}\right)^{2}}
$$


Where

$$
\begin{aligned}
& d_{i}=\min _{j, l}\left\{\sum_{k=1}^{p}\left|f_{k}\left(\boldsymbol{x}_{j}\right)-f_{k}\left(\boldsymbol{x}_{l}\right)\right|\right\} \\
& \boldsymbol{x}_{j} \in \boldsymbol{X}_{i-1}^{\prime}, \boldsymbol{x}_{l} \in \boldsymbol{X}^{\prime}-\boldsymbol{X}_{i-1}^{\prime} \quad \boldsymbol{X}_{i-1}^{\prime} \subseteq \boldsymbol{X}^{\prime}, i=1, \cdots\left|\boldsymbol{X}^{\prime}\right|
\end{aligned}
$$

The set $\boldsymbol{X}_{i-1}^{\prime}$ is a subset of the set $\boldsymbol{X}^{\prime}$, and $\boldsymbol{X}_{0}^{\prime}=\left\{\boldsymbol{x}_{1}\right\}$, $\boldsymbol{X}_{i}^{\prime}=\boldsymbol{X}_{i-1}^{\prime} \cup\left\{\boldsymbol{x}_{l}\right\}, \boldsymbol{X}_{\left|\boldsymbol{X}^{\prime}\right|}^{\prime}=\boldsymbol{X}^{\prime}, \bar{d}$ is the mean of all $d_{i}$, and $p$ is the number of objective functions.

From Definition 1 we can see that $d_{i}$ is the minimal distance between the set $\boldsymbol{X}_{i-1}^{\prime}$ and the set $\boldsymbol{X}^{\prime}-\boldsymbol{X}_{i-1}^{\prime}$. Its geometrical meaning can be seen in Figure 1.

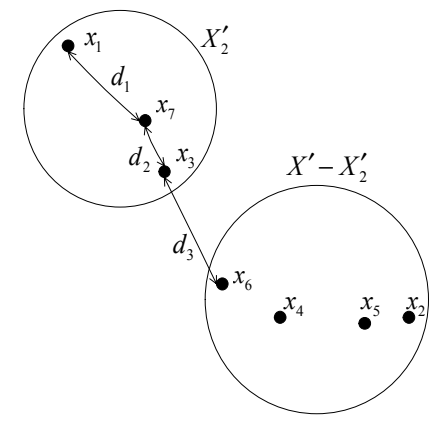

Figure 1. The geometrical meaning of $d_{i}$.

Figure 1 shows the calculating process of $d_{3}$. The minimal distance between the set $\boldsymbol{X}_{2}^{\prime}$ and the set $\boldsymbol{X}^{\prime}-\boldsymbol{X}_{2}^{\prime}$ is the distance between $\boldsymbol{x}_{3}$ and $\boldsymbol{x}_{6}$. According to the definition of $S$, its $d_{3}$ and $d_{7}$ are both the $d_{2}$ in Fig 2, so it considers one information more than once while lose some useful distribution information, such as the $d_{3}$ in Fig 2, because $d_{2}<d_{3}$ and the distance between $\boldsymbol{x}_{6}$ and $\boldsymbol{x}_{4}$ also less than $d_{3}$, so the useful information $d_{3}$ will be ignored when calculate the metric $S$. According to the definition of $\varphi$, the calculating process of $d_{i}$ is a process of constructing a connected tree. It is impossible to use one information more than once when calculate the metric $\varphi$.

Another improved metric named Nondominated Ratio is proposed in this section. The new metric $\eta$ is defined as follow.

Definition 2 (Nondominated Ratio) Let $\boldsymbol{P}_{\text {ture }}^{\mathfrak{I}}$ be a set of $\mathfrak{I}$ Paretooptimal solutions equidistantly spaced at the Pareto-optimal fronts. $\boldsymbol{P}_{\text {ag }}$ is a set of decision vectors. Then the function $\eta$ of $\boldsymbol{P}_{\text {ag }}$ is defined as equation (3):

$$
\eta\left(\boldsymbol{P}_{\mathrm{ag}}, \boldsymbol{P}_{\text {ture }}^{\mathfrak{I}}\right) \square \frac{\left|\left\{a_{\mathrm{ag}} \in \boldsymbol{P}_{\mathrm{ag}} ; \exists a_{\text {ture }} \in \boldsymbol{P}_{\text {ture }}^{\mathfrak{I}}: a_{\text {ture }} \succ a_{\mathrm{ag}}\right\}\right|}{\left|\boldsymbol{P}_{\mathrm{ag}}\right|}
$$

Where $\succ$ means Pareto Dominate, $\exists$ means inexistence.
According to Definition 2, $\eta\left(\boldsymbol{P}_{\text {ag }}, \boldsymbol{P}_{\text {ture }}^{\mathfrak{I}}\right)$ denotes the ratio between the number of the solutions in $\boldsymbol{P}_{\text {ag }}$ which are not dominated by $\boldsymbol{P}_{\text {ture }}^{\mathfrak{S}}$ and the number of all the solutions in $\boldsymbol{P}_{\mathrm{ag}}$. The metric $\eta$ measures by which each of the set of decision vectors can be assessed separately, however, we have to know the Pareto-optimal fronts when calculating it. So $\eta$ conquers the limitation of Coverage of Two Sets that must be compared between two sets. The new metrics are more intuitionistic and convenient when compared among three or more algorithms.

\section{CONCLUDING REMARKS}

Many popular test problems have been adopted to test the performances of Immune Dominance Clonal Multiobjective Algorithm [2] and SPEA2 [6]. The test results show that the Immune Dominance Clonal Multiobjective Algorithm can solve low-dimensional multiobjective problems preferably. The empirical results of the four metrics, Spacing Distribution, Nondominated Ratio, Spacing and Coverage to Two Sets, showed that Spacing Distribution conquered some limitations of Spacing, and Nondominated Ratio conquered the limitation of Coverage of Two Sets that only compared between two sets. The new metrics are more intuitionistic and convenient when compared among three or more than three algorithms.

\section{REFERENCES}

[1] Dasgupta, D., Forrest, S. Artificial immune systems in industrial applications. In IPMM '99. Proceedings of the Second International Conference on Intelligent Processing and Manufacturing of Materials. IEEE press, 1999. 257-267.

[2] Jiao, L.C., Gong, M.G., Shang, R.H., DU, H.F., Lu, B. Clonal Selection with Immune Dominance and Anergy Based Multiobjective Optimization. In Proceedings of the Third International Conference on Evolutionary Multi-Criterion Optimization, EMO 2005, Guanajuato, Mexico, 2005, 474 489.

[3] Schott, J.R. Fault Tolerant Design Using Single and Multictiteria Gentetic Algorithm Optimization. Master's thesis, Massachusetts Institute of Technology, Cambridge, Massachusetts, May 1995.

[4] Van Veldhuizen, D.A. Multiobjective Evolutionary Algorithms: Classification, Analyses, and New Innovations. $\mathrm{PhD}$ thesis. Presented to the Faculty of the Graduate School of Engineering of he Air Force Institute of Technology. Air University. USA. AFIT/DS/ENG.

[5] Zitzler, E. Evolutionary Algorithms for Multiobjective Optimization: Methods and Applications. A dissertation submitted to the Swiss Federal Institute of Technology Zurich for the degree of Doctor of Technical Sciences. Diss. Eth No. 13398. 1999.

[6] Zitzler, E., Laumanns, M. and Thiele, L. SPEA2: Improving the Strength Pareto Evolutionary Algorithm. Technical Report 103, Computer Engineering and Communication Networks Lab (TIK), Swiss Federal Institute of Technology (ETH) Zurich, Gloriastrasse 35, CH-8092 Zurich, May 2001. 